\title{
Scanning tunneling potentiometry (STP) studies of gold islands on a thin carbon film
}

\author{
J. Besold ${ }^{a}$, G. Reiss ${ }^{b}$ and H. Hoffmann ${ }^{c}$ \\ ${ }^{a}$ Institut für Festkörper- und Werkstofforschung Dresden e.V., Helmholtzstrasse 20, D-8027 Dresden, Germany \\ ${ }^{b}$ IBM T.J. Watson Research Center, P.O. Box 218, Yorktown Heights, NY 10598, USA \\ ' Universität Regensburg, Institut für Physik III, Universitätsstrasse 31, D-8400 Regensburg, Germany
}

Received 30 June 1992; accepted for publication 2 October 1992

The technique of scanning tunneling microscopy (STM) has been used to study the spatial variation of the electric potential on thin film surfaces. Topography and potential distribution of the film surface are measured simultaneously. A lateral voltage gradient is obtained by applying a DC voltage bias to a metal film. With our experimental arrangement potential differences of a few $\mu \mathrm{V}$ can be distinguished. On the lateral scale, potential drops of about $500 \mu \mathrm{V}$ can be localized within a distance of $2 \mathrm{~nm}$. On uncovered parts of the high resistive carbon film a homogeneous drop of the potential can be measured. In the vicinity of the gold islands on the carbon surface this smooth distribution is heavily disturbed. Due to their large conductance, the islands correlate well with plateaus of the measured potential. In order to identify gold and carbon on the surface, additional spectroscopic STM-measurements have been performed.

\section{Introduction}

It is of fundamental interest in solid state physics to investigate correlations between structural and electronic properties. This work tries to point out such correlations using STM experiments. The STM has been applied for few years to measure surface topography with atomic or near-atomic resolution [1,2]. There have been also some attempts to record the spatial variation of the electric potential of current carrying thin films by STP [3-5]. Both informations, topography and potential distribution of the same surface area can be used to explain surface properties of inhomogeneous and multiphase materials with local differences in the electric conductance. It is possible to study the influence of internal interfaces and individual grains in metal films and granular materials on the electric conduction process. The electrical behaviour of adsorbed or chemisorbed atoms and clusters can also be investigated in this way.
Our STM allows to measure simultaneously the surface topography and the potential distribution. Moreover we can perform spectroscopic STM-measurements. We studicd carbon films covered with an inhomogeneous gold overlayer. The gold coverage of these films is very small. There surface consists partly of carbon and partly of gold. Because there is a large difference in conductivity of gold and carbon $\left(\sigma_{\mathrm{Au}} / \sigma_{\mathrm{C}} \approx 10^{4}\right)$ STP is a very useful tool for experimental investigations of this system.

In this article we also present results concerning the sensitivity of our STP-technique. We compare the surface topography with the spatial variation of the electric potential and discuss spectroscopic STM-results of the Au/C-system.

\section{Experimental procedure}

The films were prepared by evaporation on glass substrates at room temperature. The thick- 
ness of the carbon film was $20 \mathrm{~nm}$. The gold film was grown on the carbon film surface with an average thickness of $2 \mathrm{~nm}$. On the carbon film without gold deposition $(2 \mathrm{~mm} \times 5 \mathrm{~mm})$ we measured an electrical resistance $R_{\mathrm{C}} \approx 50 \mathrm{k} \Omega$. In consequence of the gold deposition the film resistance was decreased to $20 \mathrm{k} \Omega$.

We used a room-temperature STM of the so called "Regensburger design" [2] operating in air to measure the topography. We used tungsten tunneling tips which were etched electrochemically. During the STM/STP investigation the sample-current was obtained by a floating DC voltage. An average electric field $E \leqslant 10 \mathrm{~V} / \mathrm{cm}$ was realized.

An AC tunnel voltage bias with a frequency of about $7 \mathrm{kHz}$ was applied. The DC-component of the tunneling current is kept at zero by an integrating feedback circuit. The signal of the integrator is used to determine the local potential at the position of the tip. The tip-sample distance is controlled with the rectified AC-component of the tunneling current.

\section{Results and discussion}

\subsection{Methodical investigations}

On metal films ( $\mathrm{Pt}, \mathrm{Au}$ ) and also on the $\mathrm{Au} / \mathrm{C}$-system described above, we obtained by
SIP a lateral voltage gradient corresponding with the applied electric field. Homogeneous surface areas are characterized by a linear voltage drop. Furthermore the amount of the voltage drop was constant, when we changed the direction of the applied electric field. The spatial distribution of the potential is inverted when we change the sign of the applied field. There was also no remarkable influence of the scan direction on the potential distribution. We found the same voltage gradient regardless the scanning direction was parallel or perpendicular to the electric field.

Zero point STP measurements $(E=0 \mathrm{~V} / \mathrm{cm})$ were made to find out the sensitivity of our apparatus. Simultaneously measured topography and potentiometry noise level at $E=0 \mathrm{~V} / \mathrm{cm}$ on a homogeneous area of the Au/C-system are shown in figs. $1 \mathrm{a}$ and $1 \mathrm{~b}$. The average noise level in this example is $\pm 5 \mu \mathrm{V}$. We point out that there are several contributions to this noise level. In addition to the stability of our electronic set-up also the film properties influence the noise Ievel. The spatial resolution of the potential is for example drastically decreased if the film is heated by the DC current. The integrator circuit cannot distinguish between the local potential drop caused by the applied external field and the local thermovoltage between tip and film surface. As a result of this effect, STP measurements with high spatial resolution can only be performed on films with a resistance larger than approximately $1 \mathrm{k} \Omega$.
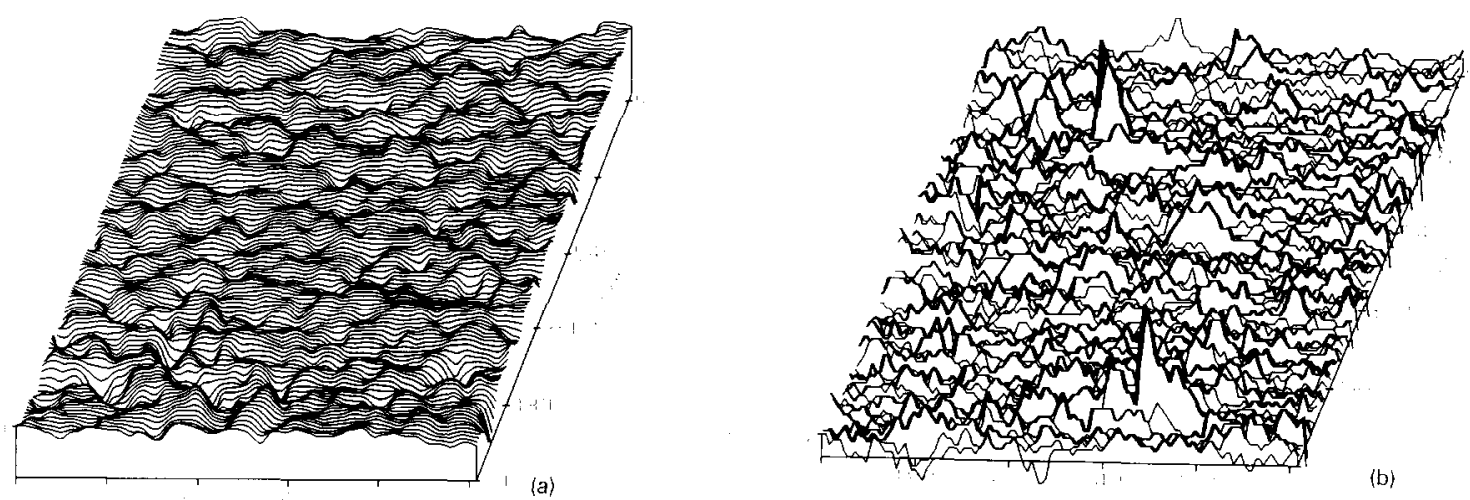

Fig. 1. (a) Topography of a homogeneous area of the $\mathrm{Au} / \mathrm{C}$-system. (b) Potential noise level of the area shown in (a) at $E=0$ $\mathrm{V} / \mathrm{cm}$. 


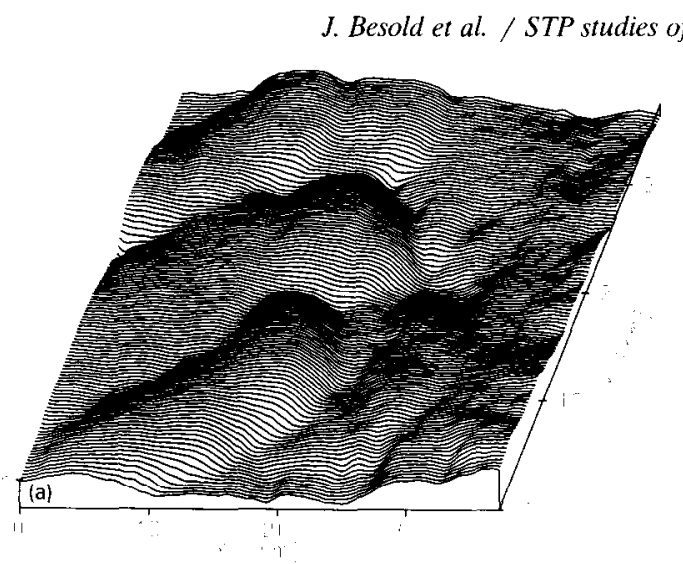

Fig. 2. (a) Topography of the Au/C-system. (b) Potential distribution of the area shown in (a), $E=10 \mathrm{~V} / \mathrm{cm}, E \|+X$.

In the case of our $\mathrm{Au} / \mathrm{C}$ system, no heating was detected up to electric fields $E \leqslant 20 \mathrm{~V} / \mathrm{cm}$.

Our integrator feedback circuit can detect very steep potential drops as shown in figs. $2 a$ and $2 b$. Though there is no distinct change in topography we can see a potential drop of about $500 \mu \mathrm{V}$ localized within a distance of $2 \mathrm{~nm}$. This voltage drop is much more than we can expect from a distance of $40 \mathrm{~nm}$ parallel to the direction of the electric field. The expected average value of the electric potential on a homogeneous surface is only $40 \mu \mathrm{V}$. We assume that the STP image in fig. $2 b$ shows a transition from a gold covered to an uncovered part of the carbon film surface which can not clearly be identified in the corresponding STM image. In further examples (figs. 3 and 4)

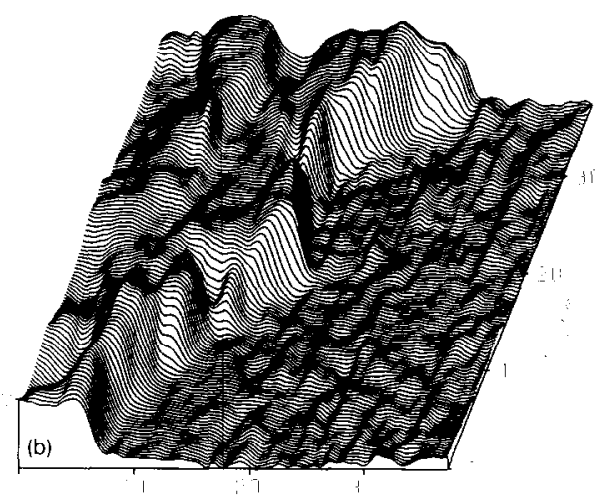

we will show, that the potential distribution on the surface of the investigated $\mathrm{Au} / \mathrm{C}$-film is very inhomogeneous due to their chemical composition.

\subsection{Study of the gold covered carbon films}

First STM/STP measurements on the uncovered carbon film surface were performed. We found a very rough surface with sharp cuts of about $10 \mathrm{~nm}$ and a potential distribution like an inclined plane with a noise level of about \pm 50 $\mu \mathrm{V}$. This large value is closely related with the high noise level of the tunneling current obtained on these carbon films. No further details can be seen in this STP image.
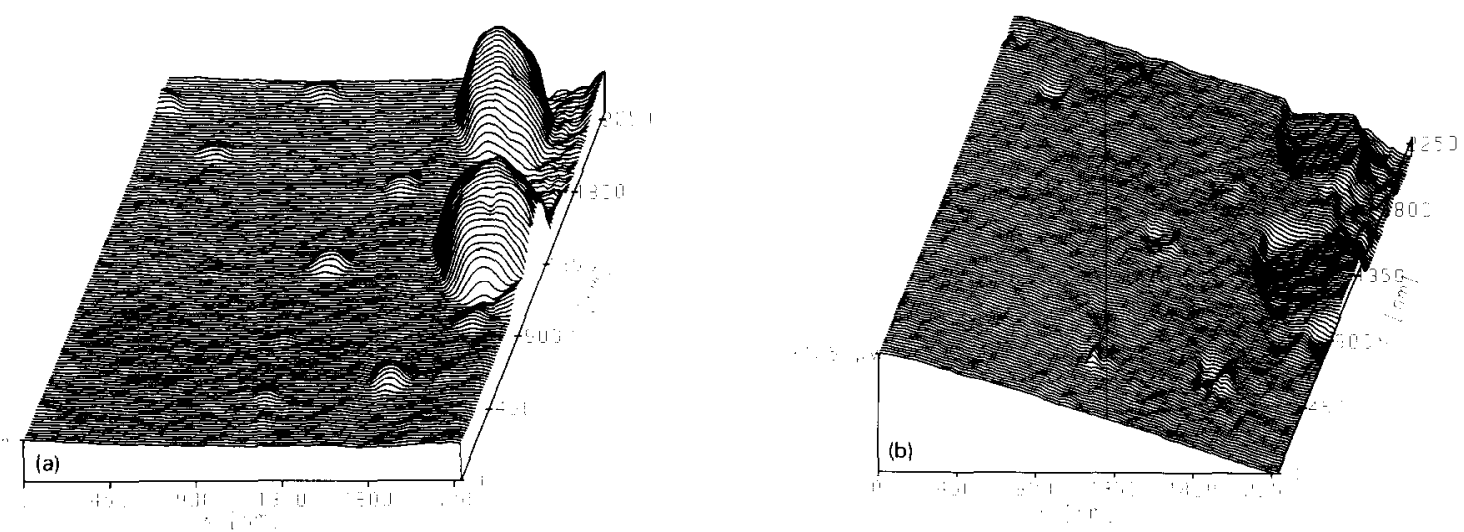

Fig. 3. (a) Topography of the Au/C-system. (b) Potential distribution of the area shown in (a), $E=10 \mathrm{~V} / \mathrm{cm}, E \|+X$. 

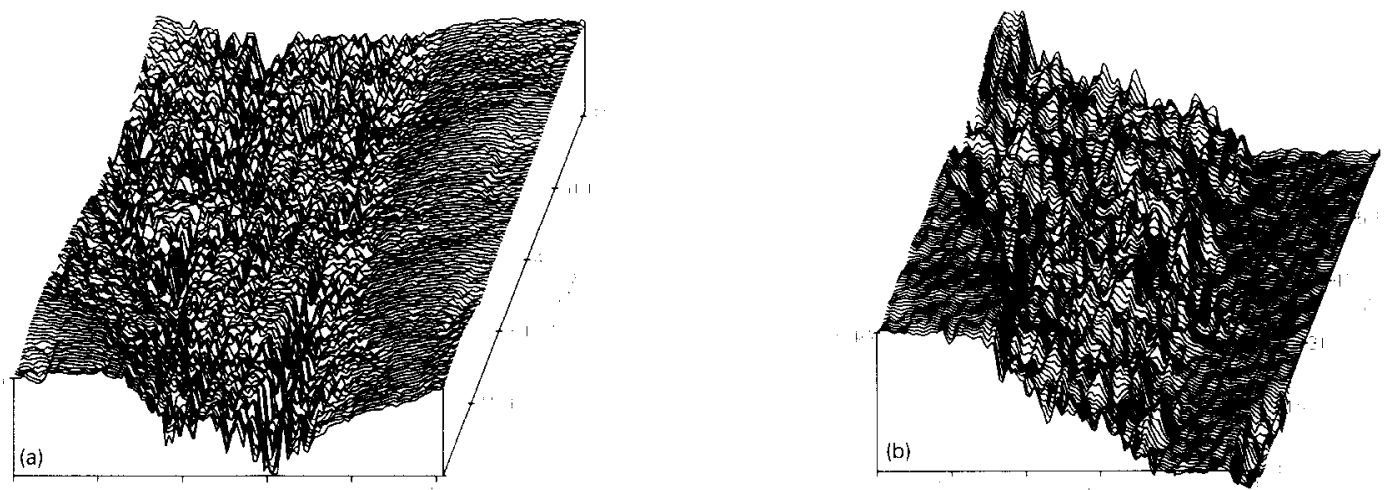

Fig. 4. (a) Topography of two neighbouring islands and the area between them. (b) Potential distribution of the area shown in (b), $E=10 \mathrm{~V} / \mathrm{cm}, E \|+X$.

Fig. 3a shows the surface topography after the gold deposition. The surface of this Au/C-system is characterized by a island like structure. The islands are surrounded by very smooth areas. We assume that gold clusters preferably in the cuts of the carbon film. The island are therefore likely consisting of gold. In the corresponding STP image shown in fig. $3 \mathrm{~b}$, we see terraces surrounded by abrupt potential drops. These plateaus correlate very well with the islands in the STM image. In contrast to this feature the smooth areas of the surface show a homogeneous drop of the electric potential. This feature can be explained by the mentioned large difference in electrical conductivity of gold and carbon. The local conductivity

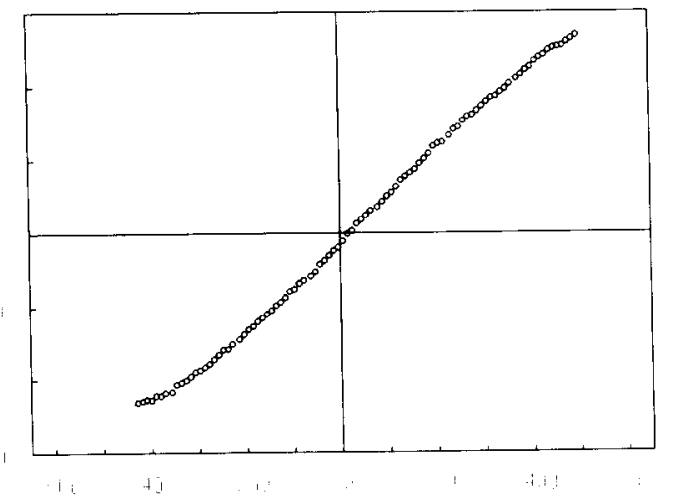

of the Au/C-film is drastically increased due the gold islands. We can also say, these islands cause local electrical short circuits. Therefore we found only a very small potential drop in the area of such an island. It seems like a plateau of nearly constant height in the spatial potential distribution. The edges of the gold islands belong to the interface between carbon and gold. The abrupt potential drop surrounding the gold islands indicates a high electrical resistance of this interface.

In figs. $4 \mathrm{a}$ and $4 \mathrm{~b}$ the topography and the potential distribution of two neighbouring islands and the area between them are shown. We see that the smooth surface of the islands correlates with plateaus in the potential distribution. These

Fig. 5. (a) $I-U$ characteristic obtained on one of the islands shown in fig. 4 a. (b) $I-U$ characteristic obtained on the area between the islands shown in fig. 4 a. 
plateaus are also characterized by a low noise level. The area between the islands is very rough and the corresponding potential distribution shows a very high noise level. We also observed a very high noise level of the tunneling current in this area similar to the uncovered carbon films. Furthermore the potential drop of about $1250 \mu \mathrm{V}$ parallel to the electric field in fig. $4 \mathrm{~b}$ is almost completely localized in the area between the island. We assume that the gold coverage in this area is very low. Therefore the potential drop is mainly caused by the two interfaces and the carbon film between the islands.

In order to identify clearly gold and carbon on the surface we have performed additional spectroscopic STM-measurements. These experiments were carried out in a constant $\mathrm{d} I / \mathrm{d} U$ mode, i.e., the feedback with the AC tunneling current was active during the $I(U)$-measurement. Figs. $5 \mathrm{a}$ and $5 \mathrm{~b}$ show $I-U$ characteristics obtained on the surface area shown in fig. 4a. In fig. 5a we see a typical measurement on one of the islands. Fig. $5 \mathrm{~b}$ shows an average $I-U$ characteristic of the area between the islands. Additionally, we performed the same spectroscopic measurement on a gold film and on an uncovered carbon film. On the gold film an average $I-U$ characteristic which is identical with fig. 5a was obtained. The $I-U$ characteristic of uncovered carbon is also comparable with fig. 5 b. We conclude from these results, that the area in fig. 4a can be clearly subdivided in two gold covered parts mentioned above as gold islands. The surface between these islands consists of carbon. We, however, additionally found on the surface of the Au/C-system gold and carbon on ncighbouring spots, too.

\section{Summary}

We developed and tested a STP technique. Using the example of gold covered carbon films, we have shown that our technique is a useful tool for the investigation of inhomogeneous surfaces of current carrying thin films. In combination with topographic and spectroscopic STM-measurements we could identify the gold islands on the carbon surface from the spatial variation of the electric potential. In further works we intend to perform STP-measurements on several materials at low temperatures. We want to investigate the metal-semiconductor-transition on metal/carbon-systems. We are also interested in the electronic behaviour of superconducting materials.

\section{Acknowledgments}

We thank J. Vancea, H. Brückl and W. Ernst for helpful discussions. This work was supported by the Deutsche Forschungsgemeinschaft (DFG).

\section{References}

[1] G. Binnig and H. Rohrer, IBM Res. Devel. 30 (1986) 355.

[2] G. Reiss, Vakuum-Technik 38 (1989) 152.

[3] P. Muralt and D.W. Pohl, Appl. Phys. Lett 48 (1986) 514.

[4] J.P. Pelz and R.H. Koch, Phys. Rev. B 41 (1990) 1212.

[5] A.D. Kent, I. Maggio-Aprile, Ph. Niedermann, Ch. Renner and O. Fischer, J. Vac. Sci. Technol. A 8 (1990) 459. 